\title{
Preparing for State Health Department Accreditation: Pivotal Roles for Public Health Surveillance
}

\author{
Christopher D. Williams* \\ DrPH Program, University of Illinois at Chicago, Baton Rouge, LA, USA
}

\section{Objective}

To specify the pertinent roles of public health surveillance in meeting documentation requirements for voluntary state health department accreditation.

\section{Introduction}

On January 24, 2014, the Public Health Accreditation Board (PHAB) released, to the public, its Standards \& Measures Version 1.5 guidance document for voluntary public health department accreditation. ${ }^{1}$ Specifically, Standard 1.2 of the guidance encompasses data collection. Two measures of Standard 1.2 stipulate the requirements of surveillance and their purposes in the context of PHAB accreditation. First, according to Measure 1.2.1A: $24 / 7$ surveillance system or set of program surveillance systems, the purpose of the measure is to assess the health department's process for collecting and managing health data for public health surveillance. Second, according to Measure 1.2.2A: Communicate with Surveillance Sites, the purpose of the measure is to assess the health department's regular contact with sites who report surveillance data to the health department. Health departments that are applying for voluntary accreditation through PHAB beginning July 1, 2014, must adhere to the current Standards \& Measures Version 1.5 guidance document. Given the recent effective date of the application period under Standards \& Measures Version 1.5, there are very few, if any, examples of required documentation that state health departments (SHDs) must submit in order to meet each public health surveillancespecific measure. Accreditation document libraries provided examples for health department applicants under Standards \& Measures Version 1.0. Because they are no longer applicable to accreditation, examples pertaining to Standards \& Measures Version 1.0 have been removed from many documentation library websites.

We specifically aim to highlight one state's informatics approach to meeting the documentation requirements of PHAB's Standards \& Measures Version 1.5, in respect to public health surveillance measures. Due to the dearth of documentation examples for PHAB's Standards \& Measures 1.5, Standard 1.2 public health surveillance measures, this perspective could be helpful to other SHDs seeking voluntary accreditation exemplifying an approach to identification of process(es) and/or protocol(s) for the collection, review, and analysis of comprehensive surveillance data on multiple health conditions from multiple sources.

\section{Methods}

When considering which documentation of process, protocol, or set of processes or protocols to include in adherence to the public health surveillance measures, ${ }^{2}$ the seven ongoing elements of any public health surveillance system can provide a roadmap. The elements are listed as follows:

Planning and Systems Design

Data Collection

Data Management and Collation

Analysis

Interpretation

Dissemination

\section{Application to Public Health Programs}

Based on the elements of a public health surveillance system and the documentation requirements of the two relevant PHAB public health surveillance measures, a documentation selection matrix of elements of a public health surveillance system and PHAB public health surveillance measure criteria is under development, and will be piloted in early November 2014. Examples of internal (within the SHD) and external surveillance systems (supportive of SHD surveillance) will be assessed using the matrix.

\section{Results}

The documentation selection matrix will be pilot tested for two weeks at the SHD. Subsequently, results will be analyzed and a summary report will be generated by the third week in November 2014. Graphical depictions of the results will be produced and made available for review.

\section{Conclusions}

An impetus for presentation at the ISDS conference is to garner feedback from attendees regarding improvement and utility of the matrix in selecting appropriate documentation to meet PHAB Standards \& Measures Version 1.5 public health surveillance measure criteria. The matrix could serve as an asset in providing tangible documentation examples for PHAB's public health surveillance measures.

\section{Keywords}

surveillance; PHAB; matrix; accreditation; SHD

\section{References}

\section{References}

Public Health Accreditation Board. (2014). PHAB standards and measures, version 1.5. Retrieved from http://www.phaboard.org/wpcontent/uploads/SM-Version-1.5-Board-adopted-FINAL-01-24-2014. docx.pdf

Thomas G. Savel, MD, Public Health Informatics and Technology Program Office, CDC, Office of Surveillance, Epidemiology, and Laboratory Services, 2500 Century Center, MS E55, Atlanta, GA 30329. Retrieved from http://www.cdc.gov/Mmwr/preview/ mmwrhtml/su6103a5.htm

*Christopher D. Williams

E-mail: cwilli52@uic.edu 